\title{
REFLECTIVE SUBCATEGORIES, LOCALIZATIONS AND FACTORIZATION SYSTEMS: CORRIGENDA
}

\author{
C. CASSIDY, M. HÉBERT AND G. M. KELLY
}

(Received 15 July 1985)

1980 Mathematics subject classification (Amer. Math. Soc.): 18 A 20.

Page 291, line 2: for $\mathscr{B}^{\perp \top}$ read $\mathscr{B}^{\top \perp}$.

Page 300, line 3: for $\left(\delta \mathcal{E}_{p i}, \Re_{o n}\right) \operatorname{read}\left(\delta \mathcal{E}_{p i}, \mathfrak{T}\right.$ on $)$.

Page 319, diagram: insert arrowheads.

Page 321, diagram: insert arrowheads.

$$
\begin{array}{ll}
\text { Page 327, diagram (9.3): } & \text { for } p_{j}^{\prime} \text { read } \rho_{J}^{\prime} ; \\
& \text { for } r^{\prime} J \text { read } r^{\prime} J .
\end{array}
$$

Passim: the punctuation following some diagrams is confusingly misplaced.

\section{References}

[1] C. Cassidy, M. Hébert and G. M. Kelly, 'Reflective subcategories, localizations and factorization systems', J. Austral. Math. Soc. (Series A) 38 (1985), 287-329.

Département de Mathématiques

Université Laval

Quebec G1K 7P4

Canada

Pure Mathematics Department

University of Sydney

N.S.W. 2006

Australia
Département de Mathématiques Université Laval Quebec G1K 7P4

Canada

(C) 1986 Australian Mathematical Society $0263-6115 / 86 \$ A 2.00+0.00$ 